\title{
The Effect of the Nova Explosion on the Evolution of Cataclysmic Variables
}

\author{
T. Naylor and M.W. Somers \\ Dept. of Physics, Keele University, Staffordshire, ST5 5BG, U.K.
}

\section{Introduction}

Classical nova outbursts are thermonuclear explosions on the surfaces of the white dwarfs in cataclysmic variables. The explosion heats the surface layers of the white dwarf, which are expected to cool on a timescale of a hundred years. The hot white dwarf should have two obvious effects on the system.

(1) It will heat the surface of the accretion disc and secondary star, increasing the overall luminosity of the system.

(2) By irradiating the surface of the secondary star it may bloat it and drive more mass transfer, thus again increasing the overall luminosity.

\section{Is the secondary star heated?}

In V1500 Cyg (Nova Cyg 1975) the heating of one side of the secondary star by the hot white dwarf causes an orbital modulation, with the brightest phase when the heated face of the secondary star is towards the observer. We have compiled measurements of the flux amplitude of the modulation from the literature. This flux amplitude declines with time, in a manner consistent with a white dwarf cooling curve (Somers \& Naylor, in prep). Thus point (1) above seems to be correct, the secondary star is heated by the cooling white dwarf.

\section{Does heating the secondary star drive a higher mass transfer rate?}

To test the second assertion in Section 1, we need to examine how the luminosity of old novae varies with time from outburst. In figure 1 we show the absolute luminosity of old novae as a function of time since outburst. The largest data set for studying the luminosity evolution is that of Vogt (1991), which we have shown as crosses. We have placed this on an absolute scale using the subset of the sample for which Cohen (1985) derived expansion parallaxes.

Obviously the older novae are the most crucial points in this diagram. Unfortunately V841 Oph was not observed prior to the peak of its outburst in 1848 . Thus the upper end of the error bar corresponds to its observed peak, the lower end to an extrapolated peak. T Sco is from Shara \& Drissen (1995).

The oldest nova is WY Sge, or Nova Sge 1783. Somers, Ringwald and Naylor (1996) detected the secondary star in this system. One can then use spectroscopic parallax to calculate the absolute magnitude. It turns out, however, that even better constraints can be derived using infrared photometry of the eclipses (Somers, Mukai \& Naylor 1996), which gives the limits shown. 


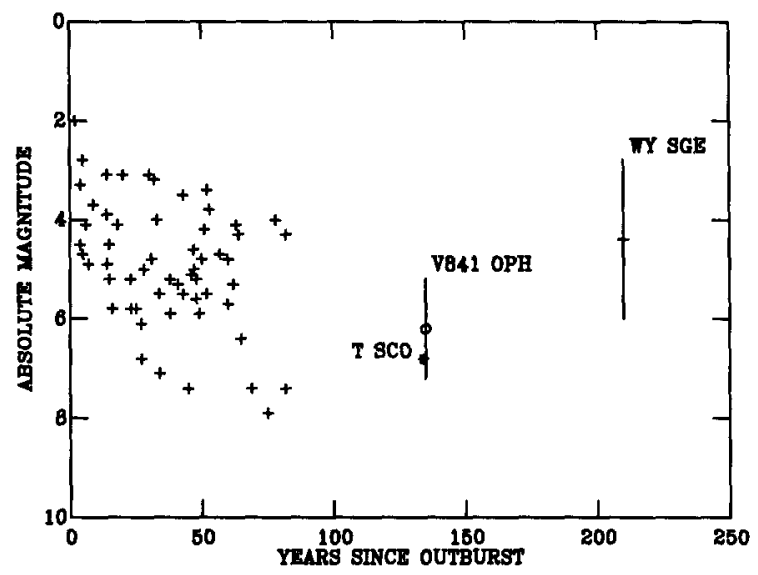

Figure 1. The luminosity of old novae vs. time since outburst.

Figure 1 shows no obvious luminosity evolution, although Vogt's data alone suggest a decline of about 2 magnitudes in the first century (Vogt, 1991). If the decline is real, it might be due to two effects.

- Ringwald, Naylor \& Mukai (1996) show that it probably takes 20 years before the nebula ceases to make a significant contribution (although the precise length of time depends on the speed class).

- There will also be a contribution from irradiation by the white dwarf.

It is clear that the irradiation does not boost the mass transfer by the large factors ( 5 astronomical magnitudes) suggested by some models (Kovetz, Prialnik \& Shara 1988). The same conclusion can be reached from the infrared lightcurves of WY Sge (Somers, Mukai \& Naylor 1996), which show that by 200 years after the outburst, the irradiation has fallen so low that it cannot be driving any mass transfer. Yet the luminosity, and hence mass transfer rate are indistinguishable from much more recent novae, where the irradiation of the secondary star will be very strong.

\section{Conclusion}

The secondary star is strongly irradiated for the first few decades after a nova outburst, but this irradiation does not drive significant mass transfer.

\section{References}

Cohen J.G., 1985, ApJ292, 90

Kovetz, A, Prialnik, D., Shara, M., 1988 ApJ325, 828

Ringwald F.A., Naylor T., Mukai K., 1996, MNRAS, 281, 192

Shara, M.M., Drissen, L., 1995, ApJ, 448, 203

Somers M.W., Ringwald F.A., Naylor T., 1996, MNRAS, accepted

Somers M.W., Mukai K., Naylor T., 1996, MNRAS, 278, 845

Vogt N., 1991, ApJ356, 609 